\title{
A SIMPLE DERIVATION OF AN APPROXIMATION TO THE TRANSIENT BLOCKING PROBABILITY IN A LOSS SYSTEM
}

\author{
M. NAIM YUNUS, ${ }^{*}$ Universiti Sains Malaysia
}

\begin{abstract}
We present a simple derivation for an approximation to the transient blocking probability of an $M / M / N$ loss system.
\end{abstract}

This approximation was initially given by Jagerman (1975). However, his derivation is fairly complicated because of the use of integral equations. In this note we derive the same approximation, but by using a much simpler method.

The approximation in question is

$$
p_{N}(t, N) \approx E_{N}(\lambda)\left[1-\exp (-(\lambda / N+1) t]^{N},\right.
$$

which approximates $p_{N}(t, N)$, the probability that at time $t$ all $N$ trunks are busy in the $M / M / N$ loss systems. This is also known as the blocking probability. $\lambda$ is the arrival rate, assuming Poisson arrivals, and $E_{N}(\lambda)$ is the Erlang loss formula. The initial condition for (1) is that all trunks are initially free, and the mean service time is normalized to 1 .

The above approximation (1) is important due to the difficulty in deriving the exact expression for the transient blocking probability in the $M / M / N$ loss system. Exact procedures are given by Riordan (1962) and Yunus (1985), but these procedures become cumbersome and complicated as $N$ increases. Approximation (1), on the other hand, is simple to use, regardless of the magnitude of $N$.

Now consider an $M / M / N$ loss system with a type of distributor that distributes the arriving calls evenly so that the rate of traffic to each trunk is $\lambda / N$. The exact blocking probability for an $M / M / 1$ loss system is easily derived, giving

$$
\tilde{p}_{1}(t, 1)=E_{1}(\lambda / N)[1-\exp (-(\lambda / N+1) t)]
$$

assuming the trunk is initially free. The tilde is used to denote that the arrival rate to this single trunk is not $\lambda$ but $\lambda / N$.

Our main assumption is

$$
p_{N}(t, N) \approx c\left[\tilde{p}_{1}(t, N)\right]^{N}
$$

where $c$ is a scaling factor, yet to be determined. We then let $t \rightarrow \infty$, that is we let the

Received 12 February 1986; revision received 2 July 1986.

* Postal address: School of Electrical and Electronic Engineering, Universiti Sains Malaysia, Jalan Bandaraya, 30000 Ipoh, Malaysia. 
system reach the steady state. Then $(3)$ becomes

$$
\begin{aligned}
E_{n}(\lambda) & \approx c\left[E_{1}(\lambda / N)\right]^{N} \\
\Rightarrow \quad c & \approx E_{n}(\lambda) /\left[E_{1}(\lambda / N)\right]^{N}
\end{aligned}
$$

since the Erlang loss formula is the steady-state blocking probability. From (3) we obtain

$$
p_{N}(t, N) \approx E_{N}(\lambda)[1-\exp (-(\lambda / N+1) t)]^{N}
$$

which is approximation (1).

To allow for any initial probability, instead of (2), we use

$$
\begin{aligned}
\tilde{p}_{1}(t, 1)= & E_{1}(\lambda / N)[1-\exp (-(\lambda / N+1) t)] \\
& +\tilde{p}_{1}(0,1) \exp (-(\lambda / N+1) t)
\end{aligned}
$$

to obtain

$$
\begin{aligned}
p_{N}(t, N) \approx & E_{N}(\lambda)[1-\exp (-(\lambda / N+1) t) \\
& \left.+\tilde{p}_{1}(0,1) \exp (-(\lambda / N+1) t) / E_{1}(\lambda / N)\right]^{N}
\end{aligned}
$$

which is the approximation for any initial probability $\tilde{p}_{1}(0,1)$ is first approximated by using (7) for a given $p_{N}(0, N)$.

\section{References}

JAGERMAN, D. L. (1975) Nonstationary blocking in telephone traffic. Bell System Tech. J. 54. 625-661.

RIORDAN, J. (1962) Stochastic Service Systems. Wiley, New York.

YuNus, M. N. (1985) Determination of time-dependent blocking probability for the $M / M / N$ loss system. Proc. 3rd. Franco-SEA Math. Conf., Kuala Lumpur. To appear. 